\title{
Electron beam irradiation processing for industrial and medical applications
}

\author{
Zehra Nur Ozer*
}

Afyon Kocatepe University, Physics Dept., Afyonkarahisar, Turkey

\begin{abstract}
In recent years, electron beam processing has been widely used for medical and industrial applications. Electron beam accelerators are reliable and durable equipments that can produce ionizing radiation when it is needed for a particular commercial use. On the industrial scale, accelerators are used to generate electrons in between $0.1-100 \mathrm{MeV}$ energy range. These accelerators are used mainly in plastics, automotive, wire and electric cables, semiconductors, health care, aerospace and environmental industries, as well as numerous researches. This study presents the current applications of electron beam processing in medicine and industry. Also planned study of a design for such a system in the energy range of $200-300 \mathrm{keV}$ is introduced.
\end{abstract}

\section{Introduction}

Irradiation process has been used in industry for many years. Electron beam irradiation is very similar to gamma radiation in terms of ionizing energy but is different in terms of dosage rates and penetration. By comparison to as gamma rays, electron beam processing has several more advantages (Table 1). Specially, electron beam technology is referred as safe and cheap technology.

Table 1. Electron beam advantages compared to gamma rays [1].

\begin{tabular}{|c|c|}
\hline Feature & Benefit \\
\hline Faster revisal & Reduced inventory \\
\hline Flexibility & Quick dose changes \\
\hline $\begin{array}{c}\text { Less material } \\
\text { degradation }\end{array}$ & $\begin{array}{c}\text { Reduced risk of product } \\
\text { damage }\end{array}$ \\
\hline $\begin{array}{c}\text { Uniform, controlled } \\
\text { dose }\end{array}$ & $\begin{array}{c}\text { Precise dose delivery with no } \\
\text { shielding from other products }\end{array}$ \\
\hline $\begin{array}{c}\text { Non radioactive dose } \\
\text { No security, transportation } \\
/ \text { on-off technology }\end{array}$ \\
\hline $\begin{array}{c}\text { Re-sourcing is not } \\
\text { required }\end{array}$ & $\begin{array}{c}\text { No radioactive cobalt resupply } \\
\text { downtime }\end{array}$ \\
\hline
\end{tabular}

Electron beam irradiation is a process for treating materials with energetic electrons that are produced by an accelerator. The electron beam accelerators cover a wide range of beam currents and energies to produce a large scale of products. Using electron beam processing, the chemical and physical properties of material is modified and the product quality is enhanced.

Energy utilization of electron beam processing is rather higher. Catalysis is not required in this system because energy is directly injected in to the material. Electron beam has directivity and high processing performance. The ratio of energy given to the material per a unit length is higher than that of gamma rays. In addition to that, the absorbed dose rate is very large since the electron beam has directivity to the direction of acceleration. Moreover, reaction control, operation and maintenance are easy for electron beam processing system. The reaction can be easily started or stopped by turning on and off the switch and by setting several parameters the system can be operated easily [2-4].

Electron beams are commonly used in industry for medical, environmental and material processing applications. In medical industry, electron beam processing system is used for medical equipment sterilization, water treatment, food preserving and pollution prevention for environmental industry [4-7]. In material processing industry, the major application of electron beam is grafting, curing, degrading and cross linking. According to the studies, $55 \%$ of the market of industrial electron beam accelerator comes from cross linking process that contains wire and cable tubing, shrink film and tires [7-10]. Here, we present a short review on the literature of industrial and medical electron beam accelerators and the important parameters in applications.

nergy and current are the two main characteristics of electron beam accelerators in industrial applications. 0.15 to $10 \mathrm{MeV}$ energy ranges of electron beams are used for processing of various applications. Low energy electron accelerators having energies 0.15 to $0.5 \mathrm{MeV}$ are generally used for treatment of surfaces and irradiation of coatings and polymeric films. Medium energetic ones produce electrons that have energies of 0.5-5 MeV. High energetic ones have energies between 5-10 MeV and they are used for sterilization of medical products and processing treatment of food.

Corresponding author: zehraerengil@,aku.edu.tr 


\section{Electron Beam Processing System}

Low energy accelerators are known as non-scanning type of electron beam processing. The source is linear source so no scanning is necessary. It is like a wide shower of electron beam to cover necessary area of the product. The medium accelerators are commonly based on transformer type and acceleration tube to produce scanned beams. In processing industry for high energy electron beam accelerators; microwave linear or radio frequency devices are used $[8,10]$.

The principal of acceleration is the basic system to produce an electron beam and accelerated them in the electron accelerator. Tungsten filament is heated at the negative cathode and electron is then emitted as a free electron called by thermionic emission. The free electron flow is accelerated by electric field and gain energy in electron volt. The accelerated electrons escape through the window and proceed towards the target material. Electrons are accelerated and given energy to be irradiated to target material by electron beam processing system. The processing system is consisting of power supply unit, accelerator tube, scanning system, irradiation window, vacuum system, product handling system and safety system.

There are different types of accelerators available with different system designs but they all use high voltage to accelerate electron in vacuum. Power supply unit is the most important part to generate high voltage of direct current. High voltage generator designed to follow the specification of the accelerator commonly based on the transformer type. The electron accelerator is usually located inside the high voltage vacuum. The main function of accelerator tube is to generate electrons and accelerate them to gain energy in electron volt. The accelerated electrons escape through the window and proceed towards the target material.

The scanning system contains focusing lens, deflection system, valve, scanning chamber, irradiation window. The beam is controlled by the focusing lenses and it is deflected through a specific angle according to design of the machine. The electron beam is allowed to be scanned sensitively for required width at target product area. Then the electron beam is applied to the material by the irradiation window. The window should be thin enough to allow electron beam to penetrate easily and reduce the heat produced by the lost electron energy while passing the window.

Vacuum system is important to prevent accelerated electron from collide with air molecules and also avoid filament to damage. The role of product handling system is to set and monitoring the quality of product. It also can shoe the status of the operation or critical process condition when there are any problems inside the machine. When high energy electron hits to the target, the $\mathrm{x}$-ray radiations are possible to generate. At this point shielding is important for accelerators. Most of the accelerators are made out of lead or steel for this purpose.

\section{Important variables for electron beam processing}

Variables are important to identify the control of the process in order to predict the production and quality of the product. These variables are:

-Absorbed dose

-Electron beam energy

-Depth of penetration

-Line speed

Absorbed dose is amount of energy that a material absorbs from processing deposited into a specific mass of material. The unit of absorbed does is in gray (Gy) usually. Absorbed dose is proportional to the ionizing energy delivered per unit mass of material. Absorbed does requirements for various industrial processes cover a wide range ( $0.1 \mathrm{kGy}$ to $1000 \mathrm{kGy}) .10 \mathrm{kGy}$ to $150 \mathrm{kGy}$ is needed for most of the applications including sterilization, polymerization, grafting, cross linking whereas less than $3 \mathrm{kGy}$ is needed to control of fungi, bacteria or parasite $[5,8,10]$.

Electron beam energy plays important role on the process. It determines how deep the energy will penetrate into the material. The penetration in the material increases linearly with the incident energy. Materials with higher electrons per unit masses have higher absorbed doses near the entrance surface but lower electron ranges. Penetration into materials of different density can be estimated by multiplying the penetration depth, found from the curves, by the ratio of the density of water to the density of the material. These curves show the penetration for different accelerating voltage to the depth of penetration in a material with mass density equal to that of water [12-14].

Line speed shows the processing rate of the material under the beam. This parameter increases with the increased beam current and decreases with the beam width and absorbed dose.

The appropriate type of industrial application depends on the electron energy and beam power requirements of the process. Electron accelerator types used for the industrial applications are high voltage direct current, microwave and radio frequency systems [10-14].

\section{Results}

Electron accelerators of several types have been developed and are being used for industrial and medical applications. Currently, there are over thousand industrial electron accelerators in operation in the world wide. These are being widely used for applications such as polymer processing, surface curing, sterilization of medical products and food irradiation.

The low energy electron accelerators can be used in coating machines, printing presses, laminating machines and so on. Medium energy electron accelerators are used to process thick plastic and rubber sheets, wire and cable insulation, plastic tubes and fiber-reinforced composites. Cross linking of wire and cable insulation is the main 
application. The higher energetic ones (up to $10 \mathrm{MeV}$ ) are usually used for sterilization, food irradiation and fine tuning of semiconductors [11-12].

Operation parameters; electron energy, beam power, irradiation width and dose rate can be precisely matched to the demands of the industrial process.

The aim of this study to give an overview of the industrial electron beam apparatuses and applications. This review on the application of electron beams in industry will help to identify potential areas in further research. We have planned to design and simulate a low energy electron curtain accelerator in low energy regime of 100-300 keV for processing the materials surface and realizing processes in gases. Further work on the design and simulation of electron accelerator is in progress.

\section{References}

1. http://ebeamservices.com/resources/advantages-of-electronbeam-over-gamma/

2. R. Mehnert, Nuclear Instruments and Methods in Physics Research B 113, (1996)

3. M. Silindir, A.Y. Ozer., FABAD J Pharm Sci 34.34 (2009).

4. N.A. Abdul Manaf, Project, University of Liverpool, Phys 379.

5. H. Lung et al., Trends in Food Science and Technology, (2015).

6. Accelerators for America's future, US Department of Energy, (2010).

7. M.R. Cleland, R.A. Galloway, New York, IBA Industrial Inc. (2009).

8. M. Kashiwagi, Y. Hoshi, Japan, NHV Corporation, Vol. 75, (2012).

9. B. Laurell, E. Föll, RadTech Europe, Switzerland, (2011).

10. M.R. Cleland, NY, USA: IBA Technology Group, (2005).

11. R.W. Hamm, M.E. Hamm, World Scientific Publishing, (2012).

12. S. Sabharwal, Proceedings of PAC2013, Pasadena, CA USA, (2013).

13. A.J. Berejka, M.R. Cleland, M. Walo, Rad. Phys. And Chem. AJB 7, (2013).

14. B. Bogdanovitch, V. Senioukov, A. Koroliov, K. Simonov, Proceedings of the 1999 Particle Accelerator Conference, NY, (1999). 